\title{
Providing Oxygen for the Crew of a Lunar Outpost
}

Frank F. Jeng and Bruce Conger Engineering and Science Contract Group

Molly S. Anderson and Michael K. Ewert NASA Johnson Space Center

\section{Space 2009}

Pasadena, California September 2009
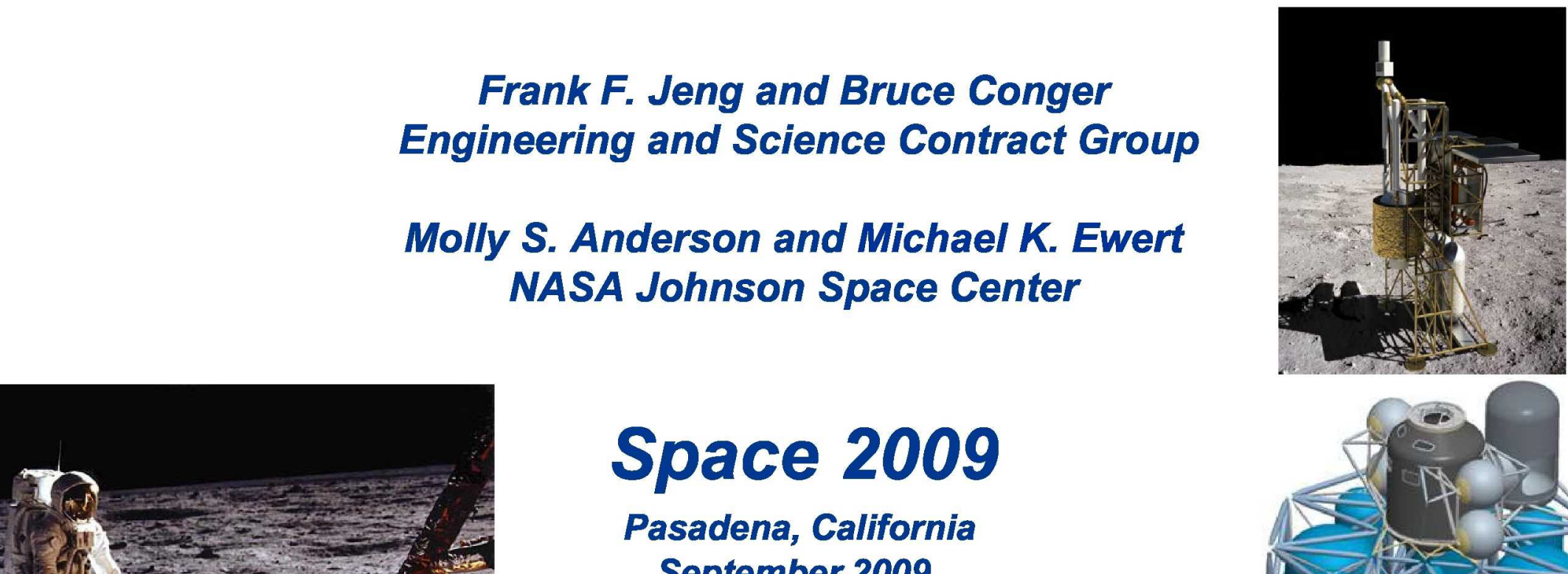

(4)
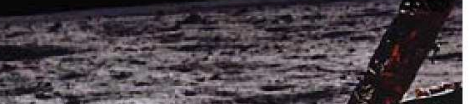

$\operatorname{lom}^{-1}$
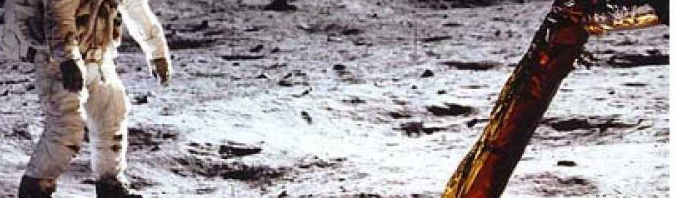

(1)
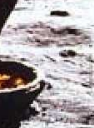

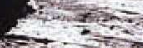
का

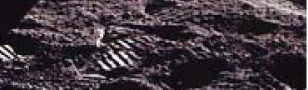

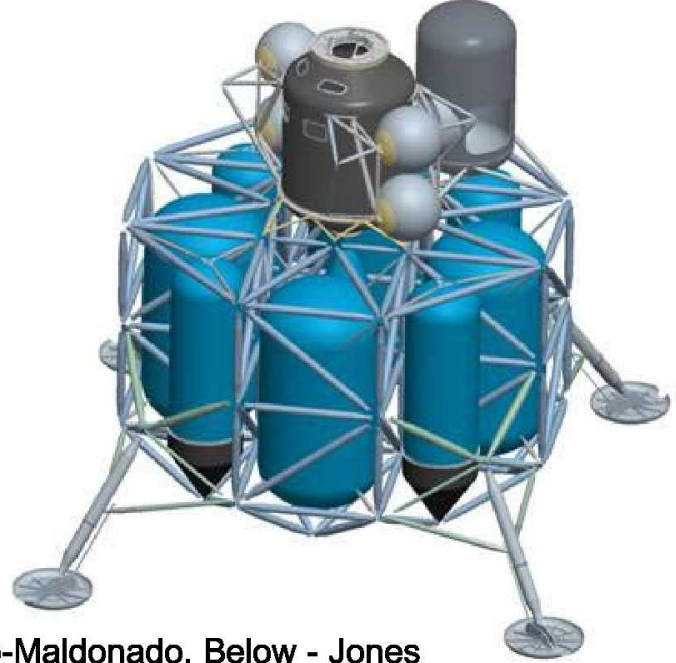




\section{Overview}

Part of a Lunar Surface Integrated Fluid Systems Analysis, which is a collaborative effort between the following Exploration Technology Development Projects:

- Exploration Life Support (ELS, a.k.a ECLSS), Extravehicular activity (EVA), In-situ resource utilization (ISRU), Propulsion and cryogenic advanced development (PCAD), Cryogenic fluid management (CFM)

- Goal: Starting with non-integrated fluid systems as a baseline, develop an optimized integrated concept and identify technology needs

This study evaluates technologies for provision of oxygen $\left(\mathrm{O}_{2}\right)$ to the crew of a Lunar Outpost

- Especially high-pressure $\mathrm{O}_{2}$ for EVA life support system recharge

- By calculating equivalent system mass (ESM) of the options

3 Cases are presented:

- 7 day Lunar Lander sortie mission

- 180 Lunar Outpost

- 10 years of successive 180 missions to the Outpost

- (Updates are included from previously reported studies) 


\section{LUNAR OUTPOST COMMON FLUIDS}

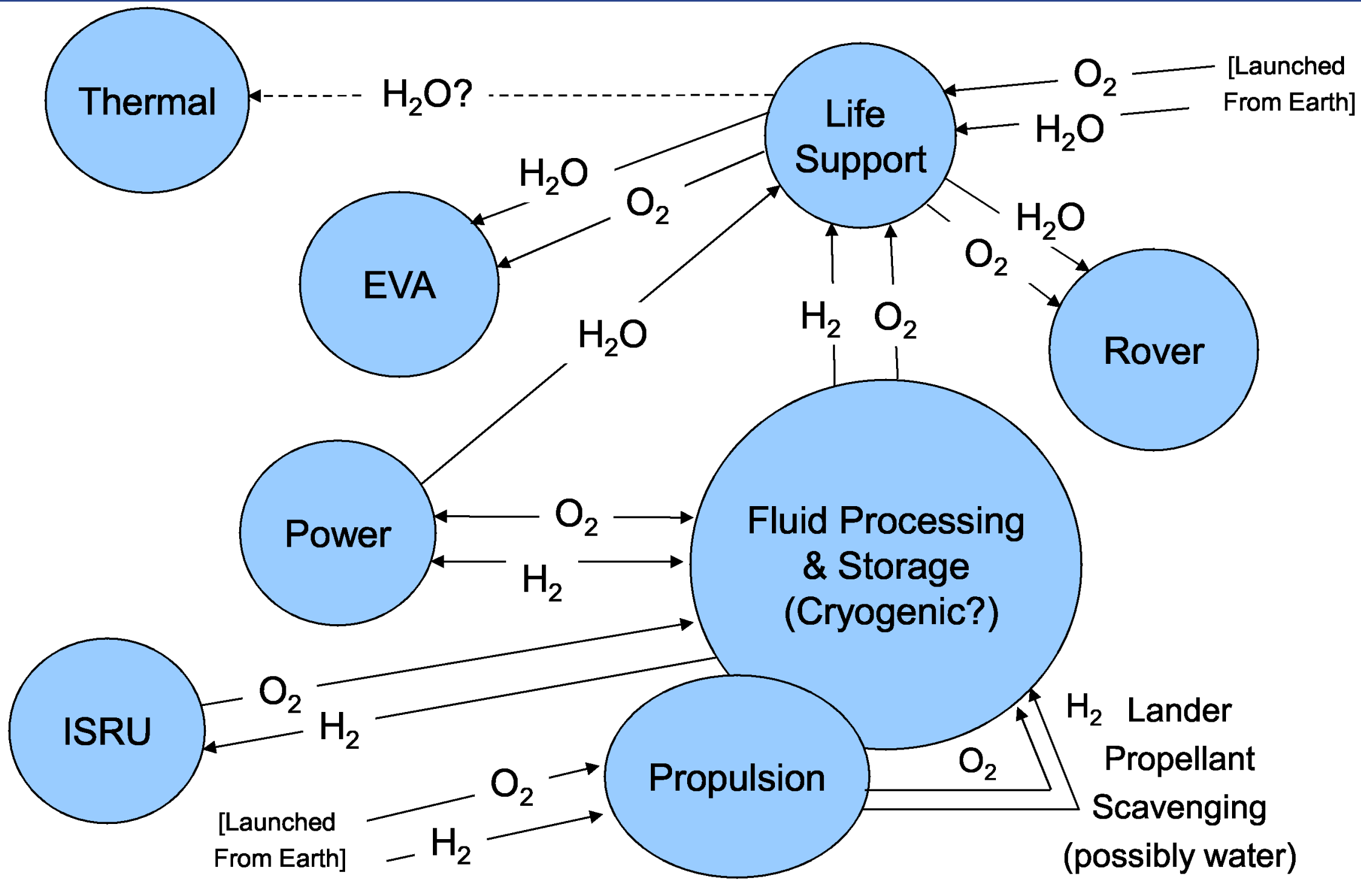




\section{Driving Requirement: High Pressure Oxygen for EVA Recharge}

Lunar exploration plan is to fill primary \& secondary oxygen bottles with one pressure at 3000 psia

Concepts for providing high pressure EVA $\mathrm{O}_{2}$ refill include:

- Store GOX in 5000 psia tanks and transfer via pressure equalization

- Low pressure electrolysis followed by mechanical compression

- Store LOX in space suit to provide for breathing, $\mathrm{CO}_{2}$ washout \& thermal control (results shown in previous paper)

- Convert LOX into high pressure oxygen by:

direct heating and expansion for EVA and ECLSS applications (a.k.a. "vaporizer")

Or by using a Temperature Swing Adsorption Compressor (TSAC)

Use left over LOX in propellant tanks if available; otherwise, bring it along

- High pressure electrolysis

May be able to share the electrolyzer of the regenerative fuel cell at an Outpost

- Compress and store GOX from ISRU

4 person crew

- 2 crew per EVA (if 4 crew per EVA is required, double these results) 


\section{Crew Requirements for Oxygen (180 day case)}

Current Shuttle/ISS EMU $\mathrm{O}_{2}$ bottle capacities are:

- Primary $\mathrm{O}_{2}, 850$ psia

- Secondary $\mathrm{O}_{2,}, 6000$ psia
$1.2 \mathrm{lb}$

$2.6 \mathrm{lb}$

High pressure oxygen users in Lunar Outpost

- $\mathrm{O}_{2}$ for $\mathrm{EVA}^{3}$

Primary $\mathrm{O}_{2}$ bottle, 3000 psia

Secondary $\mathrm{O}_{2}$ bottle, 3000 psia (infrequent)

Suit purge, $<20$ psia

High pressure (3000 psia) $\mathrm{O}_{2}$ resupply (primary tank only)

Low pressure metabolic $\mathrm{O}_{2}$ consumption

Number of Outpost EVA Sorties

- Nominal EVA-CM hours ${ }^{2}$ (8 hours/CM-EVA)

- 300 CM-EVA's in 180-day Outpost mission

Total $\mathrm{O}_{2}$ requirements (high and low pressures)

Total high pressure $\mathrm{O}_{2}$ required (180 days)

- Mission average delivery rate

- Design $\mathrm{O}_{2}$ daily delivery rate (consecutive day EVAs)

$1.6 \mathrm{lb} / \mathrm{CM}^{1}$-EVA

$2.6 \mathrm{lb} / \mathrm{CM}-E V A$

$1.0 \mathrm{lb} / \mathrm{CM}-E V A$

$1.6 \mathrm{lb} / \mathrm{CM}-\mathrm{EVA}$

$1.94 \mathrm{lb} /$ day

2400 hours

$1745 \mathrm{Ib}$

$480 \mathrm{lb}$

$2.7 \mathrm{Ib} /$ day

$3.2 \mathrm{lb} / \mathrm{day}$

$\square$ 3. Conger, B., Falconi E., Greg Leavitt, and Chullen C.," PLSS Baseline Schematics and Internal Interfaces”, Rev. A, JSC-65563/CTSD-CX-5117 


\section{Baseline Technology - High Pressure Oxygen Tanks}

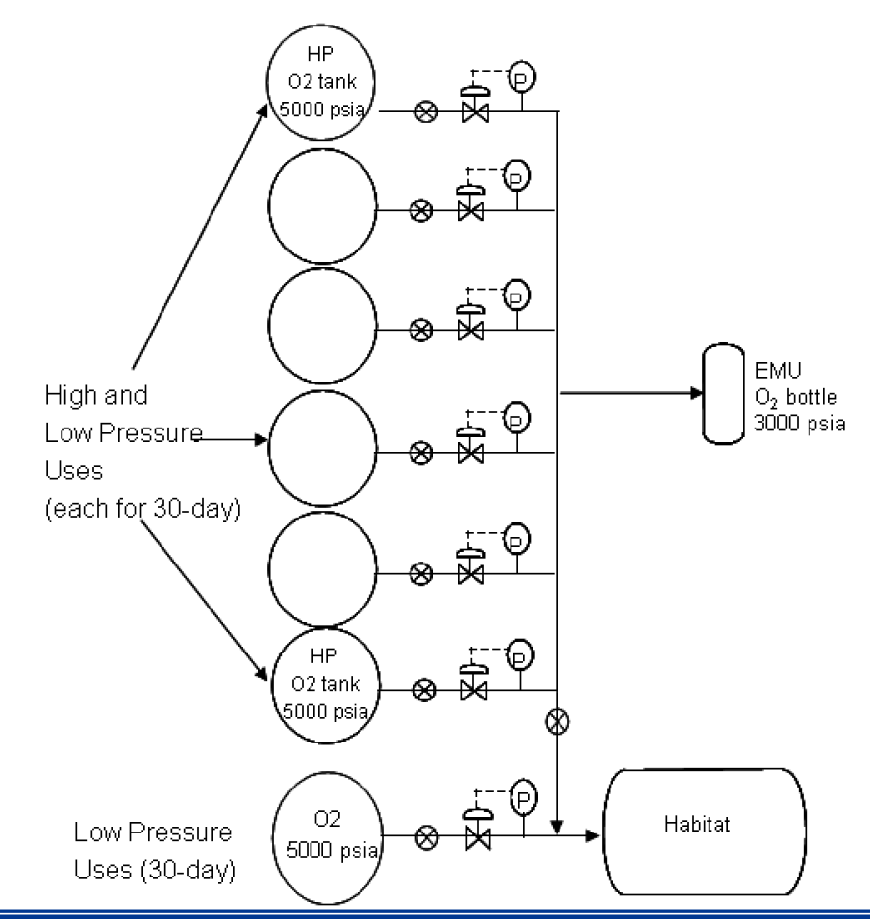

Simple and reliable

Tanks made of Incone ${ }^{\circledR}$ liner over-wrapped with carbon-fiber are relatively light weight.

For oxygen gas storage, 5000 psia is a reasonable limit.

- Material compatibility and safety issues increase with higher pressures

While 3100 - 3600 psia is the range for charging EVA oxygen bottles, left-over oxygen is enough for medium/low pressure applications, such as:

Crew breathing

EMU purging

Other ECLSS use 


\section{Low Pressure Water Electrolyzer, Dryers and Multi-Stage Piston Compressor}

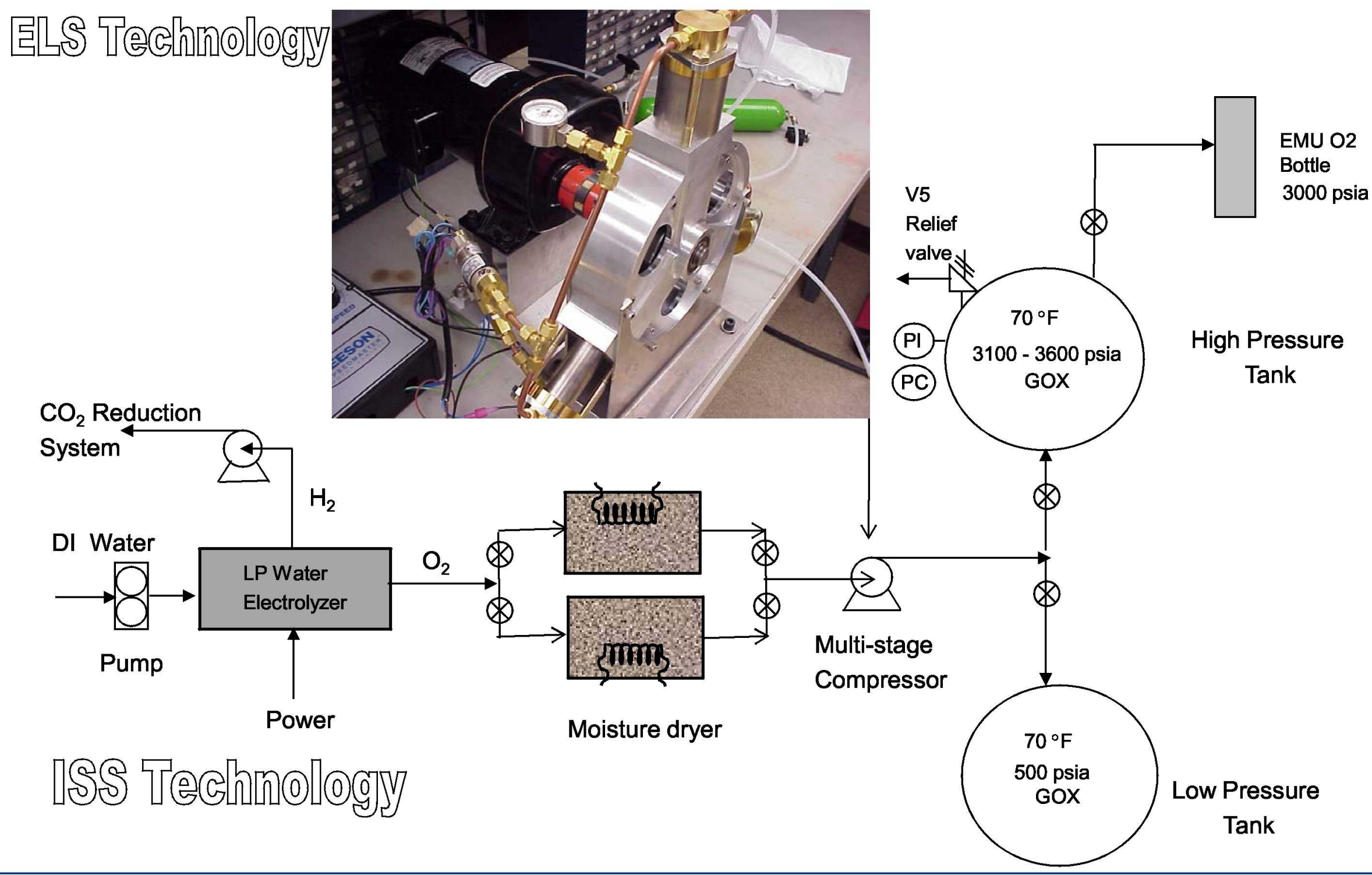




\section{Deliver LOX to HP Tank and Vaporize to High Pressure (Supply from Dedicated Tanks or Propellant Scavenging)}

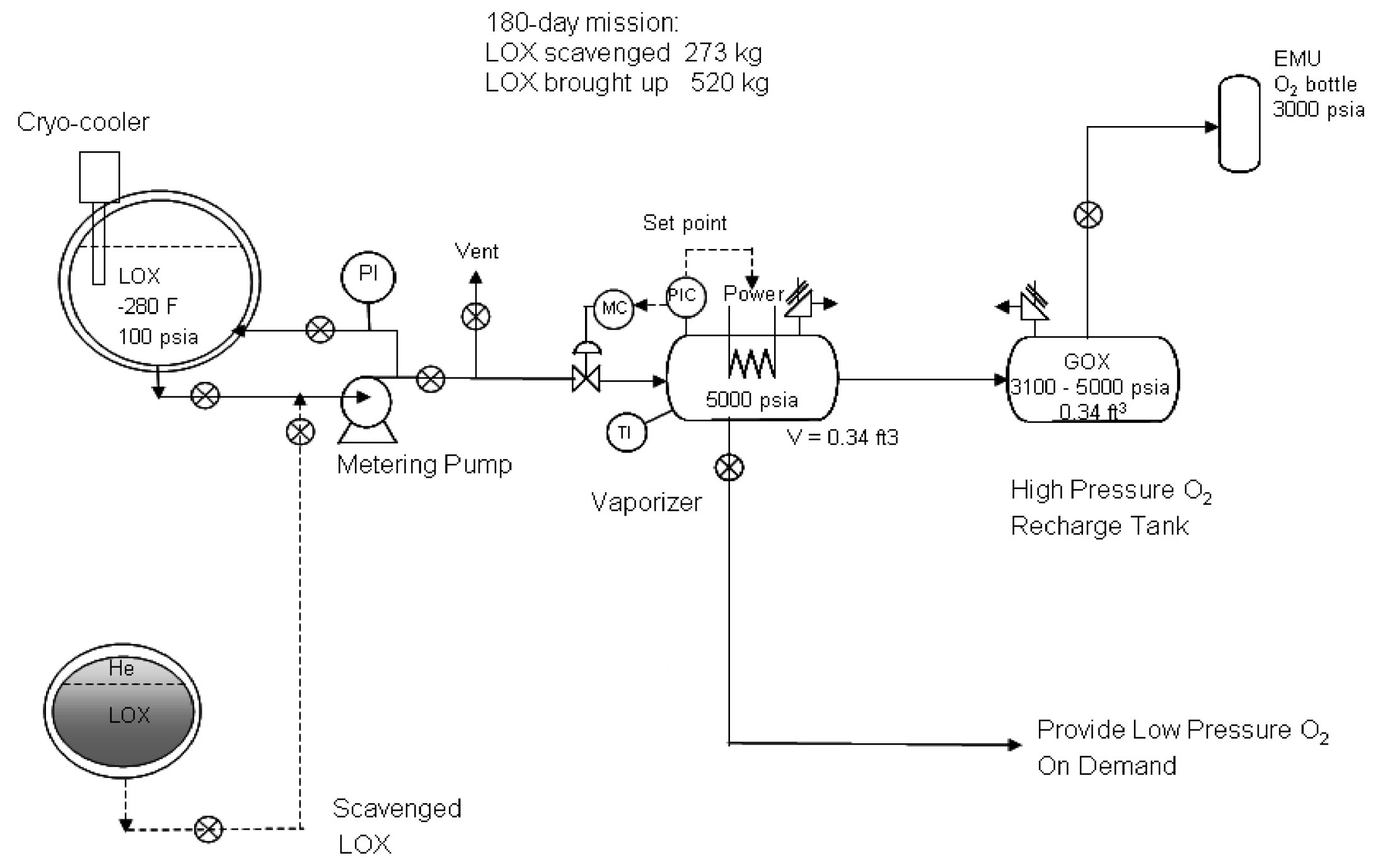

Thermal energy on lunar surface is used to generate high pressure GOX, heater is for back-up. 


\section{Cryo Tank, Thermal Swing Adsorption Compressor and Additional Compressor}

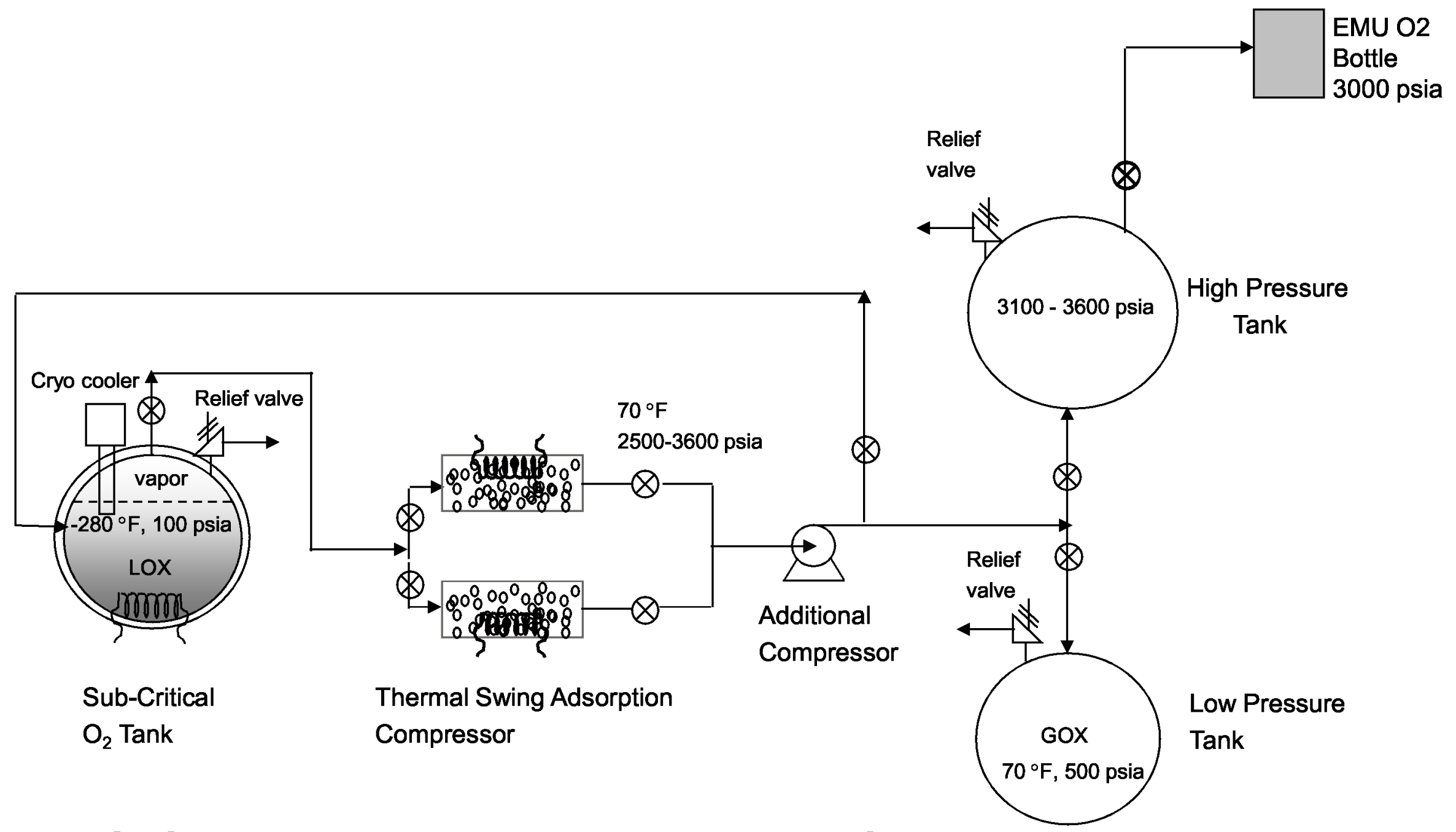

The TSAC is solid-state technology; its operation life should be longer than a mechanical compressor; but lower TRL 


\section{Generating High Pressure $\mathrm{O}_{2}$ Using Fuel Cell and High Pressure Electrolyzer}
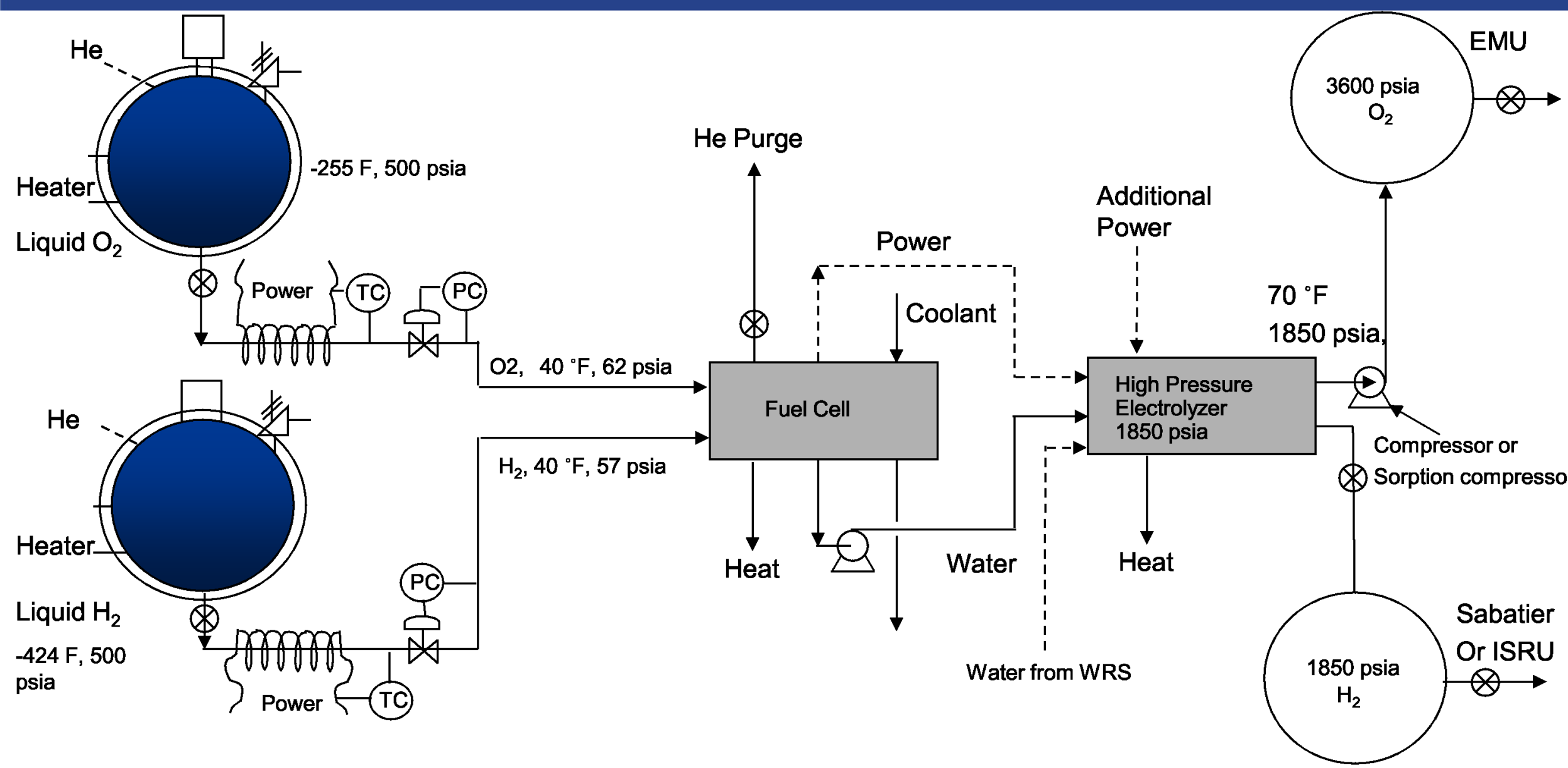

Solid Polymer Electrolysis (SPE®) water electrolysis technology has been used in UK and US submarines for approximately 20 years

- Hamilton Sundstrand has accumulated 50,000+ hours in testing a single cell water electrolysis unit operated at 1850 psia

Lunar Surface Power System and ECLSS could share the high pressure water electrolyzer

Two options for generating $\mathrm{O}_{2}$ at 3600 psia:

- Use an electrolyzer operating at 3600 psia

- Use a mechanical compressor to boost $\mathrm{O}_{2}$ pressure from 1850 psia to 3600 psia (assumed here) 


\section{Import Moderate Pressure 02 from ISRU and Compress to Desired EVA $\mathrm{O}_{2}$ Pressure}

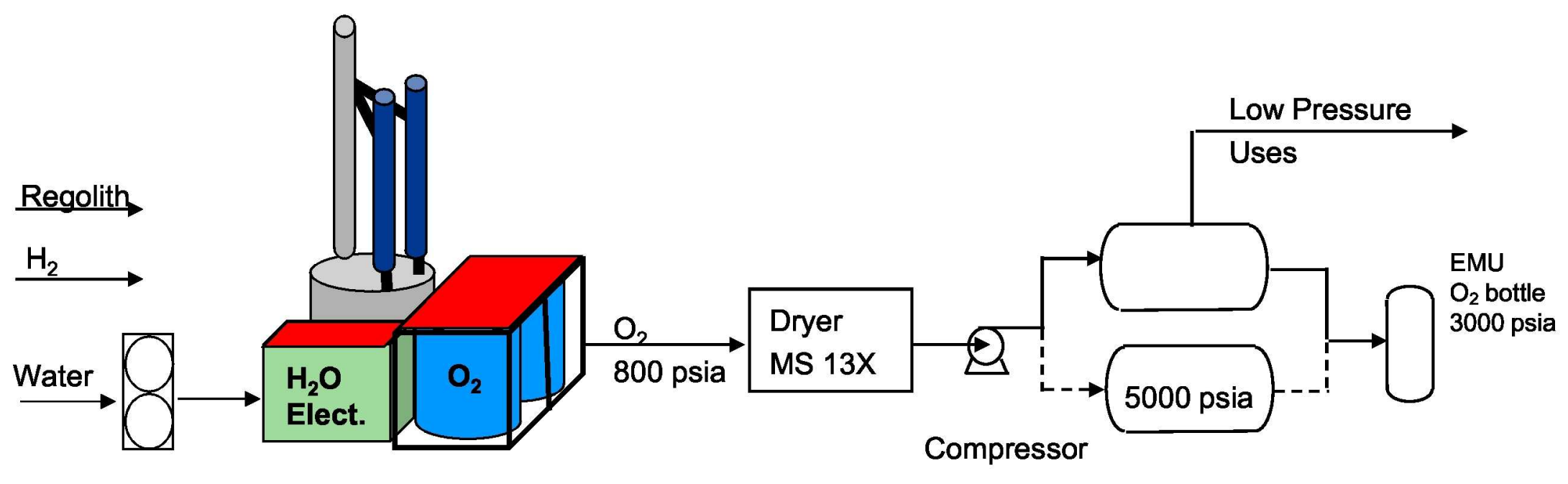

Pump

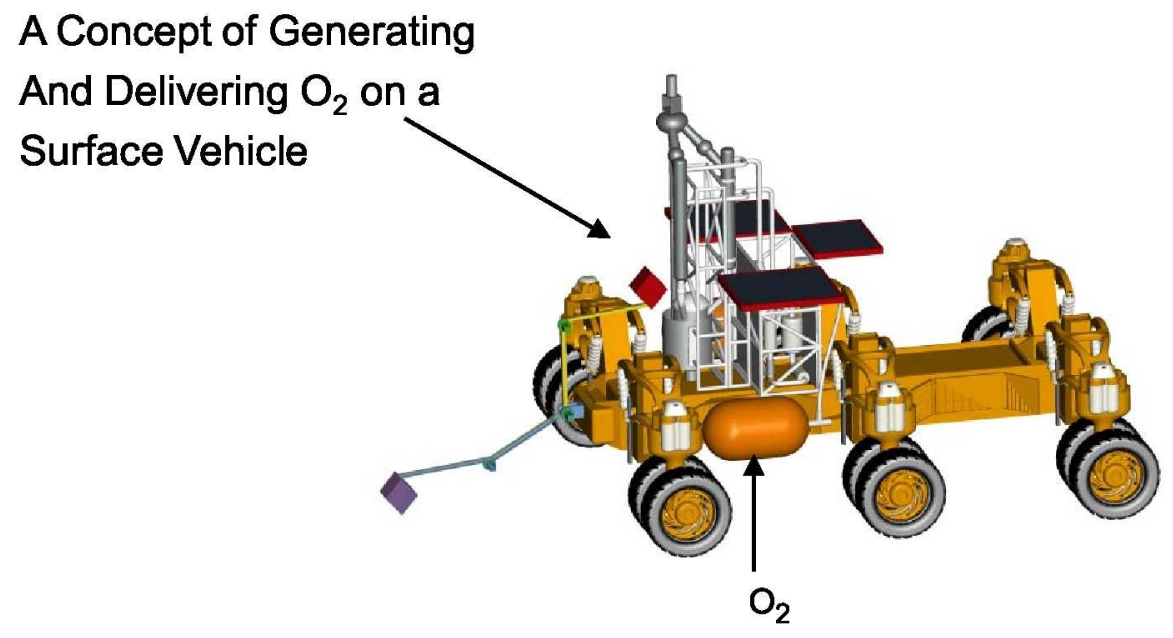




\section{Mass and ESM of $\mathrm{O}_{2}$ Generation Options (Open architecture) - Lunar Lander 7-day Sortie Mission}

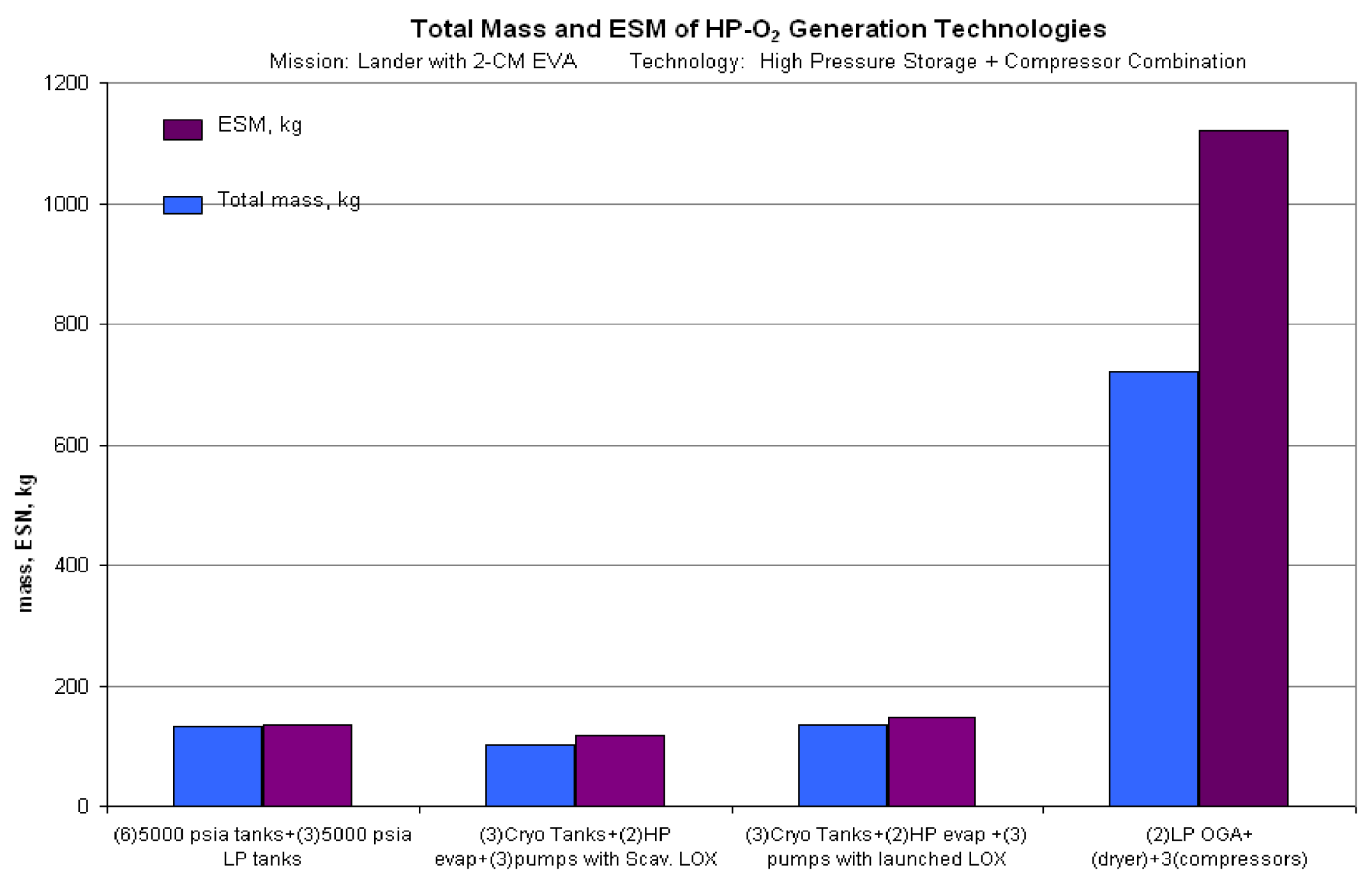




\section{Mass and ESM of $\mathrm{O}_{2}$ Generation Options (Open architecture) - 180-day Mission}

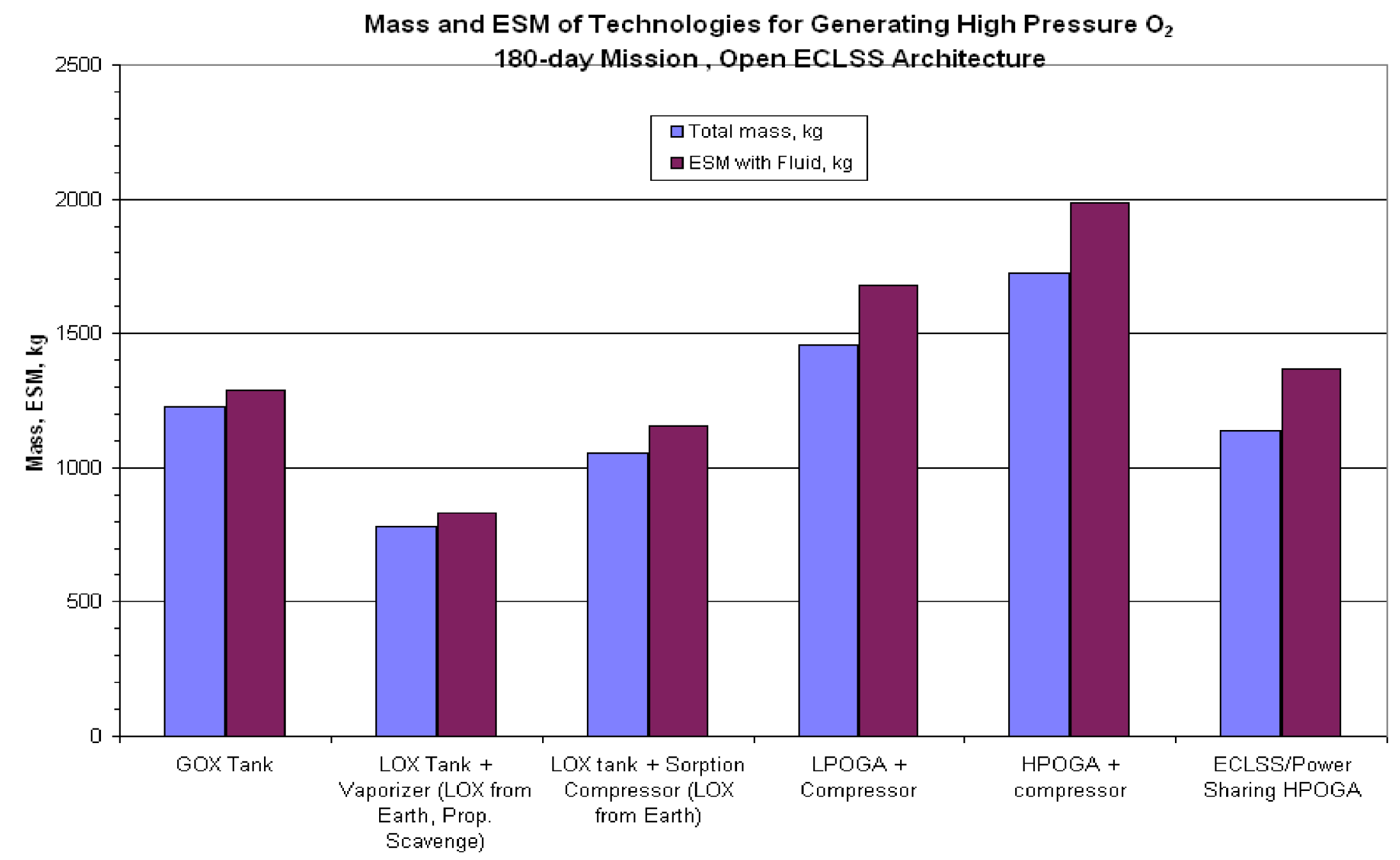




\section{Mass and ESM of $\mathrm{O}_{2}$ Generation Options (Closed architecture) - 10-year Outpost Mission}

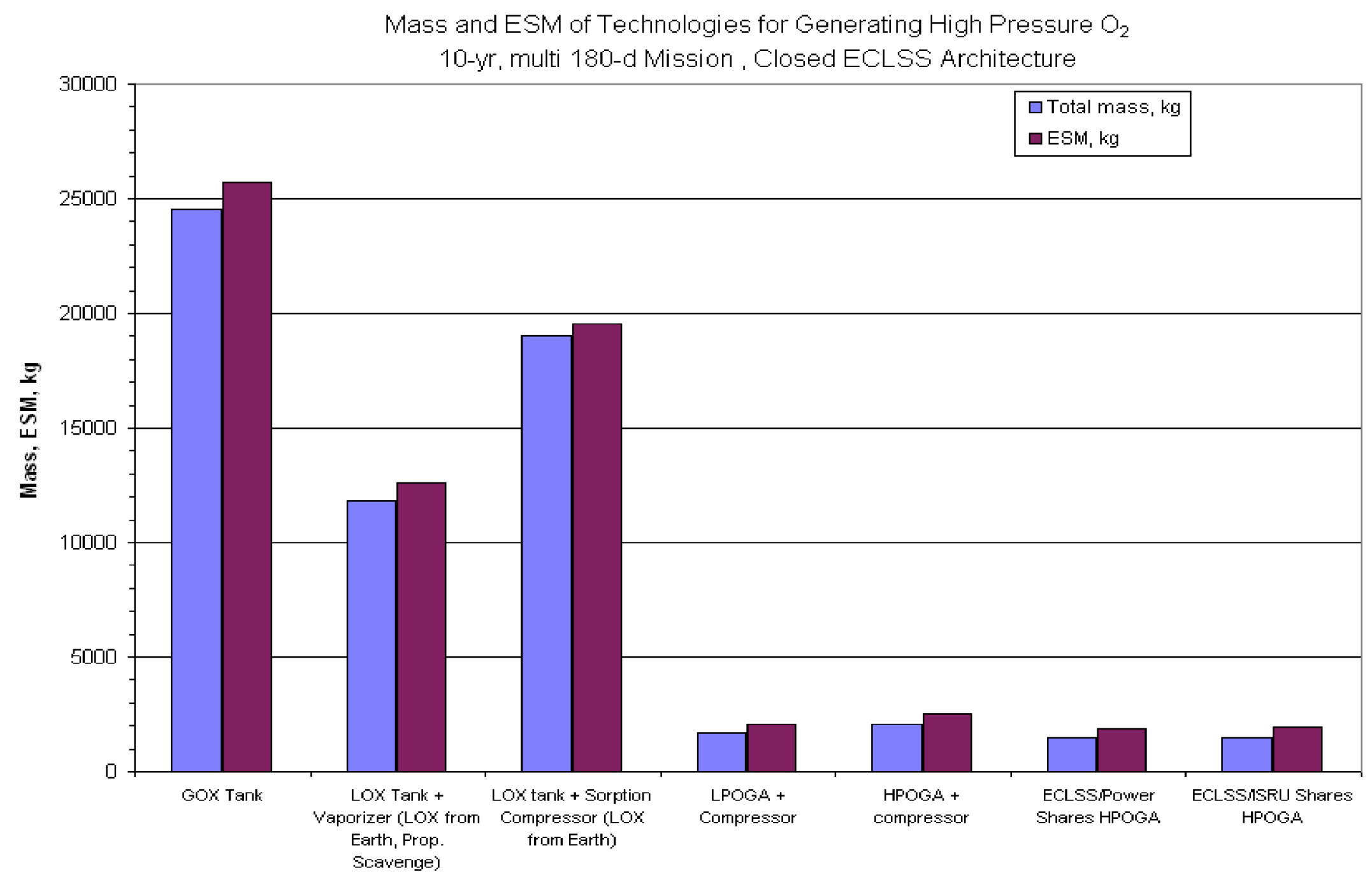




\section{Conclusions and Recommendations}

For Sortie missions (open ECLSS architecture)

- Scavenging LOX \& vaporizing it to meet high/low pressure $\mathrm{O}_{2}$ needs has lowest ESM, followed closely by launching 5000 psia GOX tanks

For a180-day Outpost mission (open ECLSS architecture)

- Scavenging LOX \& vaporizing it into high/low pressure GOX has lowest ESM, followed by LOX plus sorption compressor

For 10-yr multi 180-day missions (closed ECLSS architecture)

- Sharing high pressure oxygen generation assembly (HPOGA) between Power and ECLSS has the lowest total mass and ESM

- But the ISRU option \& low pressure electrolysis with compressor are within 10\% of HPOGA

Development of the following technologies recommended:

- Multi-stage $\mathrm{O}_{2}$ piston compressor

- Vaporization and compression of a LOX/GOX mixture to 3600 psia

A thermal compression technology with few rotating components

- High pressure cryogenic $\mathrm{O}_{2}$ pump

An essential component in the above LOX vaporizing technology

- HPOGA for generating $\mathrm{O}_{2}$ up to 3600 psia and $\mathrm{H}_{2}$ to a moderate pressure.

A technology shared among Power, ECLSS and ISRU Elements.

Further Study recommended on:

- Risks, including micrometeoroids and high pressure oxygen safety in the various options

- Optimization of exact $\mathrm{O}_{2}$ tank pressure and geometry

- The propellant scavenging concept, including launching extra $\mathrm{O}_{2}$ for delivery of $\mathrm{LOX}_{\text {or }} \mathrm{H}_{2} \mathrm{O}$

- Mission water balance

- Mission scenarios involving pressurized rovers 


\section{References}

Jeng, Frank, et al., "High Pressure Oxygen Generation for Lunar Outpost EVA", SAE 2009-01-2534, SAE 39th International Conference on Environmental Systems, Savannah, GA, July 12-16, 2009 Jeng, Frank, "High-Pressure Oxygen Generation for Lander EVA Study", ESCG-4470-09-TEAN-DOC-0100, ESC Group, Houston, TX, July 30, 2009. Jeng, Frank, "High-Pressure Oxygen Generation for Outpost EVA Study", ESCG-4470-08-TEAN-DOC-0358A, ESC Group, Houston, TX, November 24, 2008.

Jeng, Frank, "Status of Integrated Lunar Fluid Systems Analysis", ESCG4470-09-TEAN-DOC-0039", ESC Group, Houston, TX, March 31, 2009.

Jones, Brad. "Altair (Lunar Lander) Project Overview." Presentation to AIAA Space 2008 Conference \& Exposition. San Diego, CA. September 10, 2008.

Linne, D.L., et al., "Feasibility of Scavenging Propellants from Lander Decent Stage to Supply Fuel Cells and Life Support", AIAA-2009-6511, AIAA Space 2009, Pasadena CA, September 14-17, 2009.

Polsgrove, Tara, et al. "Altair Lunar Lander Consumables Management", AIAA-2009-6589, AIAA Space 2009, Pasadena, CA, September 14-17, 2009. 


\section{Backup Material}




\section{Mass, Volume, Power \& ESM for $\mathrm{O}_{2}$ Provision (Sortie Mission) - 7-d Sortie Mission}

\begin{tabular}{|l|c|c|c|c|c|c|}
\hline & $\begin{array}{c}\text { Oxygen/ } \\
\text { Water, } \\
\mathrm{kg}\end{array}$ & $\begin{array}{c}\text { Mass } \\
\mathrm{kg}\end{array}$ & $\begin{array}{c}\text { Volume, } \\
\mathrm{m}^{3}\end{array}$ & $\begin{array}{c}\text { Power, } \\
\text { W }\end{array}$ & $\begin{array}{c}\text { Cooling, } \\
W\end{array}$ & ESM, kg \\
\hline $\begin{array}{l}\text { GOX Tanks } \\
\text { (5000 psia) }\end{array}$ & 34 & 132 & 0.106 & 0 & 0 & 136 \\
\hline $\begin{array}{l}\text { Cryo Tanks + } \\
\text { Evaporizers + } \\
\text { Scavenged LOX }\end{array}$ & 0 & 102 & 0.190 & 42 & 4 & 116 \\
\hline $\begin{array}{l}\text { Cryo Tanks + } \\
\text { Evaporizers + } \\
\text { Launched LOX }\end{array}$ & 32 & 134 & 0.190 & 42 & 4 & 149 \\
\hline $\begin{array}{l}\text { LP OGA + } \\
\text { Compressors }\end{array}$ & 40 & 721 & 0.457 & 1,610 & 889 & 1,121 \\
\hline
\end{tabular}

Notes:

1. Includes mass of oxygen/water and hardware. 


\section{Mass, Volume, Power \& ESM for $\mathrm{O}_{2}$ Provision (Open Architecture) - 180-d Mission}

\begin{tabular}{|l|c|c|c|c|c|c|c|}
\hline Technologies & $\begin{array}{l}\text { Fluid, } \\
\mathrm{kg}\end{array}$ & $\begin{array}{l}\text { Hardware } \\
\text { Mass, } \mathrm{kg}\end{array}$ & $\begin{array}{l}\text { Volume, } \\
\mathrm{m}^{3}\end{array}$ & $\begin{array}{l}\text { Power, } \\
W\end{array}$ & $\begin{array}{l}\text { Cooling } \\
W\end{array}$ & $\begin{array}{l}\text { Total } \\
\text { Mass, } \mathrm{kg}\end{array}$ & $\begin{array}{l}\text { ESM of } \\
\text { Hardware and } \\
\text { Fluid, } \mathrm{kg}\end{array}$ \\
\hline GOX Tank (5000 psia) & $891^{1}$ & 337 & 2.314 & 0 & 0 & 1,228 & 1,288 \\
\hline $\begin{array}{l}\text { LOX Tank+ Vaporizer } \\
520^{2}\end{array}$ & 264 & 1.715 & 19 & 7 & 784 & 830 \\
\hline $\begin{array}{l}\text { LOX Tank+ Sorption } \\
\text { Compressor }\end{array}$ & $804^{3}$ & 373 & 1.844 & 444 & 432 & 1,052 & 1,155 \\
\hline $\begin{array}{l}\text { LP OGA + } \\
\text { Compressor }\end{array}$ & $894^{4}$ & 560 & 1.285 & 1,703 & 982 & 1,454 & 1,682 \\
\hline $\begin{array}{l}\text { HP OGA + } \\
\text { Compressor }\end{array}$ & $894^{4}$ & 832 & 1.596 & 1,999 & 1,006 & 1,726 & 1,985 \\
\hline $\begin{array}{l}\text { ECLSS/Power } \\
\text { Sharing HP OGA }\end{array}$ & $894^{4}$ & 243 & 0.947 & 2,012 & 1,019 & 1,137 & 1,368 \\
\hline
\end{tabular}

\footnotetext{
Notes:

1. Includes $\mathrm{O}_{2}$ left over in the tank.

2. $520 \mathrm{~kg}$ LOX launched from Earth. No mass penalty for $\mathbf{2 7 3} \mathrm{kg}$ scavenged from Lander propellant

3. All LOX from Earth.

4. Mass of water for electrolysis.
} 
Mass, Volume, Power and Resupply Penalties of $\mathrm{O}_{2}$ Generation Options (Closed Architecture) - 10-yr Outpost Mission

\begin{tabular}{|c|c|c|c|c|c|c|c|c|}
\hline Technologies & $\begin{array}{l}\text { Initial } \\
\text { Mass } \\
\text {, } k g\end{array}$ & $\begin{array}{l}\text { Initial } \\
\text { Volume, } \\
m^{3}\end{array}$ & $\begin{array}{l}\text { Power } \\
\text {, }\end{array}$ & $\begin{array}{l}\text { Cooling, } \\
W\end{array}$ & $\begin{array}{l}\text { Resupply } \\
\text { Mass, } k g\end{array}$ & $\begin{array}{l}\text { Resupply } \\
\text { Volume, } \\
M^{\wedge} 3\end{array}$ & $\begin{array}{l}\text { Total } \\
\text { Mass }^{1}, \mathrm{~kg}\end{array}$ & $E S M, \mathbf{k g}$ \\
\hline GOX Tanks & 1,227 & 2.314 & 0 & 0 & 23,319 & 43.966 & 24,546 & 25,740 \\
\hline LOX Tanks + Vaporizer & 784 & 1.715 & 19 & 7 & 11,025 & 29.054 & 11,809 & 12,605 \\
\hline $\begin{array}{l}\text { LOX Tank + Sorption } \\
\text { Compressor }\end{array}$ & 1,052 & 1.715 & 444 & 432 & 17,994 & 17.299 & 19,047 & 19,573 \\
\hline $\begin{array}{l}\text { LP OGA + } \\
\text { Compressor }^{2}\end{array}$ & 459 & 0.464 & 1,703 & 982 & 1,232 & 1.151 & 1,691 & 2,088 \\
\hline $\begin{array}{l}\text { HP OGA + } \\
\text { Compressor }^{2}\end{array}$ & 733 & 0.775 & 2,003 & 1,010 & 1,482 & 1.482 & 2,080 & 2,516 \\
\hline $\begin{array}{l}\text { ECLSS/Power Sharing } \\
\text { HP OGA }\end{array}$ & 137 & 0.126 & 2,012 & 1,019 & 1,347 & 1.482 & 1,484 & 1,905 \\
\hline $\begin{array}{l}\text { ECLSS/ISRU Sharing } \\
\text { HP OGA }\end{array}$ & 161 & 0.396 & 2,074 & 1,081 & 1,346 & 1.482 & 1,507 & 1,943 \\
\hline
\end{tabular}

1. Total mass Includes fluid and hardware.

2. Includes $73 \mathrm{~kg}$ make-up water for each 180-day mission with a closed ECLSS architecture. 


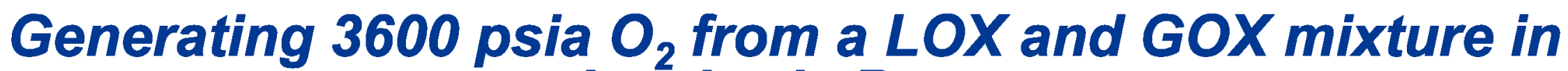 an Isochoric Process}
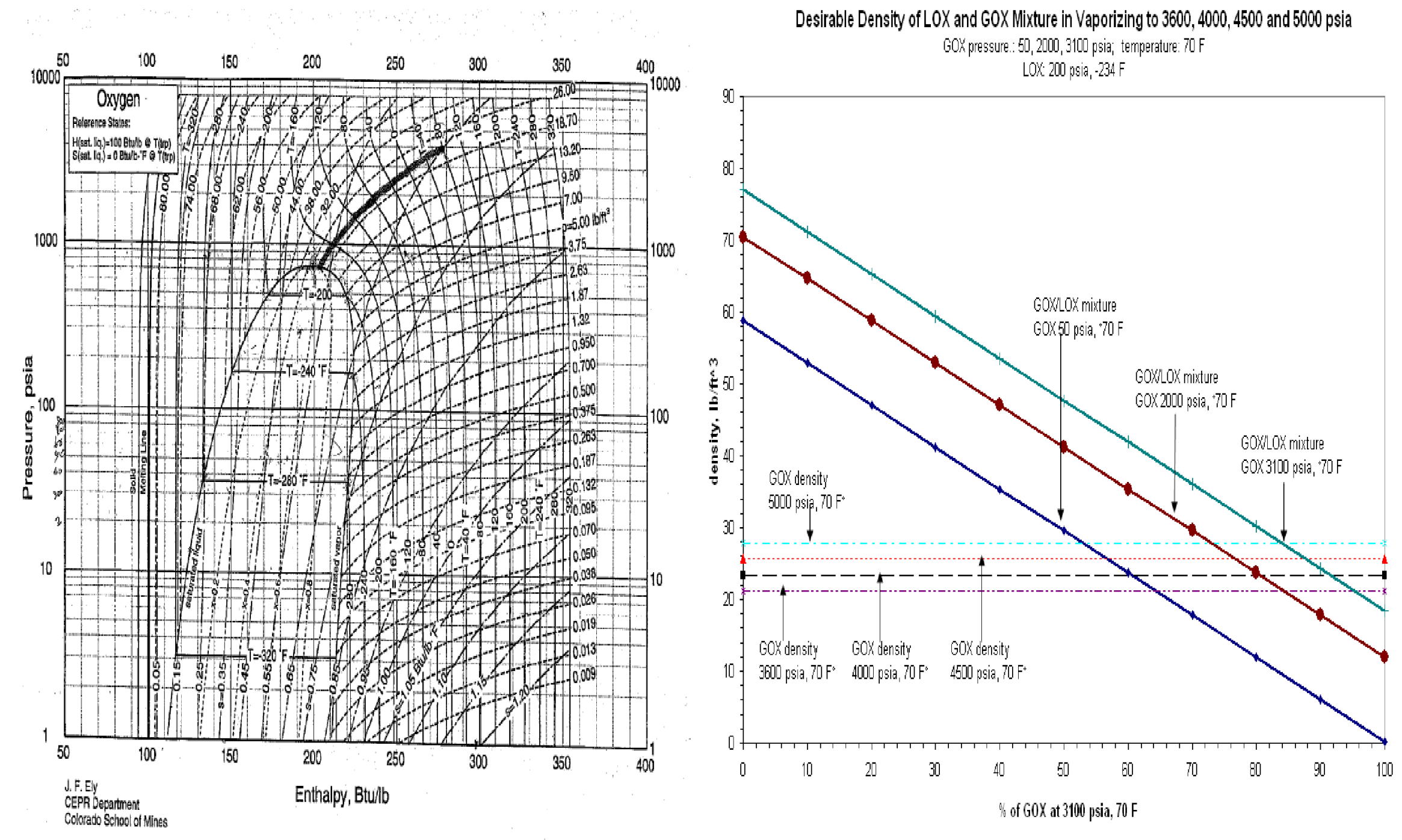